procedures seemed to be possible, and moreover, in these 3 groups, more than $80 \%$ of cases harbored the hematomas as large as $3-6 \mathrm{~cm}$ in diameter or more.

\title{
36. Experimental Studies on Intracerebral Hemorrhage
}

\author{
Haruyuki Kanaya, Seiya Sirakata, Yasuhiro Kuzu, Kanzi Saotome, \\ Seizi Sinmaru, Humio Nomura, Setsuo Ono and Kazuo Kozima \\ II Department of Surgery, Iwate Medical College
}

The clinicopathological changes in the early stage in experimental intracerebral hemorrhage, which was made by putting the fresh clot into internal capsule, were studied. Results were obtained as follows:

1) Blood pressure rised slightly and puls rate showed no changes or decreased. Respiration became rapid immediately after putting and slow lately.

2) Cerebrospinal fluid pressure rised immediately 2 or 3 times as normal and maintained in high pressure.

3) Cerebral blood flow (carotic artery) and $\mathrm{O}_{2}$ volume of cerebral blood decreased.

4) Cerebral oxygen tention decreased immediatly and recovered in 30 to 60 minutes and then increased afterward.

5) $\mathrm{O}_{2}$ and $\mathrm{CO}_{2}$ volume of periferal blood showed no marked changes, but $\mathrm{O}_{2}$ volume of venous blood tended to decrease.

6) Leucocyte count did not show any constant changes and blood sugar decreased, and $\mathrm{Ht}$, serum protein showed no changes.

From the above results, it may be concluded that cerebral blood circulation and cerebral metabolism are markedly decreased.

\section{The Operation of Hypertensive Intracerebral Hemorrhage}

\author{
Takao Mitsuno, Haruyuki Kanaya, Seiya Shirakata, Yasuhiro Kuzu, \\ Kanzi Saotome and Seizi Shinmaru \\ II Department of Surgery, Iwate Medical College
}

Twenty cases of massive intracerebral hemorrhage out of 38 surgically treated cases of cerebral apoplexy, including 3 cerebral softenings, were operated upon from 12 hours to 13 days after stroke. And though 18 cases had been complicated 